\title{
Deterministic chaos and fractal entropy scaling in Floquet conformal field theories
}

\author{
Dmitry S. Ageev,,${ }^{1, *}$ Andrey A. Bagrov, ${ }^{2,3, \dagger}$ and Askar A. Iliasov ${ }^{4,5, \$}$ \\ ${ }^{1}$ Steklov Mathematical Institute, Russian Academy of Sciences, Gubkin Street 8, Moscow 119991, Russia \\ ${ }^{2}$ Department of Physics and Astronomy, Uppsala University, Box 516, SE-75120 Uppsala, Sweden \\ ${ }^{3}$ Theoretical Physics and Applied Mathematics Department, Ural Federal University, Mira Str. 19, 620002 Ekaterinburg, Russia \\ ${ }^{4}$ Institute for Molecules and Materials, Radboud University, Heyendaalseweg 135, NL-6525 AJ Nijmegen, The Netherlands \\ ${ }^{5}$ Space Research Institute of the Russian Academy of Science, Moscow 117997, Russia
}

(Received 27 July 2020; revised 3 March 2021; accepted 8 March 2021; published 26 March 2021)

\begin{abstract}
In this Letter, we study two-dimensional Floquet conformal field theory, where the external periodic driving is described by iterated logistic or tent maps. These maps are known to be typical examples of dynamical systems exhibiting the order-chaos transition, and we show that, as a result of such driving, the entanglement entropy scaling develops fractal features when the corresponding dynamical system approaches the chaotic regime. For the driving set by the logistic map, the fractal contribution to the scaling dominates, making entanglement entropy a highly oscillating function of the subsystem size.
\end{abstract}

DOI: 10.1103/PhysRevB.103.L100302

The concept of Floquet driving keeps attracting considerable interest in different areas of quantum many-body theory since it provides a powerful approach to the controllable manipulation of the phases of matter. In the study of topologically protected states of matter [1,2], many-body localization [3,4], and thermalization $[5,6]$, it was shown to be a tool for both externally tuning conventional phase transitions [7] and engineering novel phases that are nonexistent in equilibrium [8].

A natural playground to address the dynamics of periodically modulated correlated systems is the framework of Floquet conformal field theories (CFTs). Recently, a wide class of Floquet modulated two-dimensional (2D) CFTs has been proven to be exactly solvable [9-11]. In the proposed setting, one drives the system by periodically interchanging two Hamiltonians, namely, the canonical Hamiltonian of 2D CFT on a strip, and its deformation. While originally the particular case of the so-called sine-square deformation (SSD) has been considered $[9,12]$, later on, it was realized that the dynamics of inhomogeneous CFT [13-16] is exactly solvable for general deformations $[17,18]$. Since already in the simplest SSD case, the driving leads to nontrivial energy flows in the system causing the localization of energy density, whose pattern resembles black-hole-like structures $[10,11,19]$, it is tempting to exploit the approach in its full generality and investigate what types of unconventional emergent phenomena can occur in Floquet driven systems.

\footnotetext{
*ageev@mi-ras.ru

†andrey.bagrov@physics.uu.se

†a.iliasov@science.ru.nl
}

Published by the American Physical Society under the terms of the Creative Commons Attribution 4.0 International license. Further distribution of this work must maintain attribution to the author(s) and the published article's title, journal citation, and DOI. Funded by Bibsam.
To proceed along this line, one should bear in mind that the Floquet theory as a branch of mathematics can be viewed as a part of a more general theory of classical dynamical systems [20-22]. In previous works $[9,10]$, the following correspondence between driven two-dimensional conformal field theories and dynamical systems has been established implicitly:

\section{Evolution of a deformed 2D CFT}

\section{企}

Holomorphic dynamics of iterated maps

Dynamical systems defined by the iterative composition of simple maps are known to exhibit chaos, bifurcations, and self-organization phenomena. For many standard choices of the holomorphic maps, the evolution of the corresponding dynamical system generates fractals. Fractals, which are objects of noninteger dimension, have intriguing nonsmooth behavior and can appear even in the dynamics of seemingly regular structures. A canonical example of a fractal is the Julia set that can emerge as the spectrum of quasiperiodic (the famous Hofstadter butterfly) or self-similar potentials [23-25]. In fact, the emergence of fractals [26] is commonplace for a general choice of iterated functions. Many studies are devoted to understanding fractal structures in cold-atom systems, topological insulators, mesoscopic systems, etc. [27-34].

Treating the Floquet driving of a CFT as a dynamical system, which can manifest chaotic or fractal properties on the level of map iterations, does not evidently correspond to the common approach normally taken in quantum chaos theory $[35,36]$. Hence it is interesting to elucidate the connection between chaotic dynamics in quantum systems and the more classical theory of dynamical systems. In this Letter, we report that periodic Floquet modulation based on a certain choice of iterated holomorphic maps can evoke fractal patterns of quantum correlations in the driven state. Namely, it leads to the emergence of an exotic class of quantum states with 
a fractal scaling law of entanglement entropy. To achieve that, we consider CFT deformations that can be described in terms of dynamical systems generated by either logistic or tent maps. Both these maps are extremely simple: The tent map is a discontinuous linear function and the logistic map is a quadratic function. Still, they lead to highly nontrivial dynamics exhibiting a transition to the regime of deterministic chaos, irregular dynamics governed by deterministic laws that are highly sensitive to the initial conditions [37].

Following the proposal of Refs. $[9,38]$, we start with a theory defined on the strip of width $L$ in the complex plane,

$$
H=\int_{0}^{L} \frac{d x}{2 \pi}[f(w) T(w)+f(\bar{w}) \bar{T}(\bar{w})],
$$

where $w=\tau+i x, x \in(0, L) .{ }^{1}$ Here, $T(w)$ and $\bar{T}(\bar{w})$ are the holomorphic and antiholomorphic components of the stress-energy tensor correspondingly, and $f(w)$ defines the deformation, with $f(w)=1$ corresponding to the undeformed CFT.

To introduce the Floquet driving, we first perform a conformal map from the strip onto the complex plane with a slit by

$$
z=\exp \left(\frac{2 \pi}{L} w\right)
$$

and then make change of variables $\chi=\chi(z)$,

$$
\chi(z)=\exp \left(\int \frac{d z}{z f(z)}\right),
$$

bringing (1) to the uniform Hamiltonian [10],

$$
H=\frac{2 \pi}{L} \int_{\mathcal{C}} \frac{\chi}{2 \pi i} T(\chi) d \chi+\text { c.c. }+\cdots,
$$

where a constant term resulting from the transformation is omitted. In these coordinates, the Hamiltonian evolution in Euclidean time $\tau$ is given simply by the dilatation transformation $\chi \rightarrow \lambda \chi$ with $\lambda=e^{\frac{2 \pi}{L} \tau}$ (throughout the rest of this Letter, we take $L=1$ ). In $z$ coordinates, this implies that the evolution in Euclidean time for some $\tau$ governed by the deformed Hamiltonian (1) reduces to a change of variables $z_{1}(z)$ obeying the identity

$$
\chi\left(z_{1}\right)=\lambda \chi(z),
$$

which is the Schröder spectral functional equation from the theory of dynamical systems [39]. The Hamiltonian evolution for time $n \tau$ is then given by the composition

$$
z_{n}(z)=\underbrace{\left(z_{1} \circ z_{1} \cdots \circ z_{1}\right)}_{n \text { times }}(z) .
$$

From this, it readily follows that the Floquet driving of a CFT, when the system Hamiltonian is periodically swung with time step $\tau$, can be described by alternating the composition of two different functions $z_{1}(z)$ and $\widetilde{z}_{1}(z)$,

$$
z_{n}(z)=\underbrace{\left(z_{1} \circ \tilde{z}_{1} \cdots \circ z_{1}\right)}_{n \text { times }}(z)
$$

\footnotetext{
${ }^{1}$ After an analytical continuation, $x$ would be the spatial coordinate.
}

where each of the functions corresponds to its own deformation $f(z)$.

We will be working with two dynamical systems defined by the logistic map

$$
z_{1}^{\log }(x)=\alpha x(1-x)
$$

and the tent map

$$
z_{1}^{\text {tent }}(x)=\left\{\begin{array}{l}
\beta x, \quad x<\frac{1}{2}, \\
\beta(1-x), \quad x>\frac{1}{2},
\end{array}\right.
$$

which can also be equivalently represented as

$$
z_{1}^{\text {tent }}(x)=\frac{\beta}{\pi} \arcsin [\sin (\pi x)],
$$

where $x \in(0,1) .^{2}$ The Floquet evolution of the CFT is then given by iterated composition (6) of maps (8) or (9) and the dilatation. For both choices, it can be shown that by properly rescaling the holomorphic map, the stroboscopic dynamics can in fact be reformulated as a Hamiltonian dynamics following a single quench [39].

The iterative dynamics of these maps gradually becomes more and more complicated when the $\alpha$ and $\beta$ parameters approach the critical values $\alpha_{\text {chaotic }}=4$ and $\beta_{\text {chaotic }}=2$ corresponding to the truly chaotic regime. One can naturally expect that properties of a CFT driven by such a dynamical system would encode the deterministic chaos in a nontrivial way. To study that, for the Floquet driving Eq. (7), we choose $z_{1}$ to be either the tent or logistic map, and $\widetilde{z}_{1}$ the dilatation transformation.

As shown in the Supplemental Material [39], in order to describe the evolution of a CFT with some deformation $f$, a one-step transformation $z_{1}$ should allow the Schröder equation (5) to have nontrivial solutions. $f(z)$ can then be restored from (3). In general, it is hard to find the solution for $\chi$ analytically, and the answer is known only for a limited number of functions $z_{1}[40,41]$. In the chaotic regime, for the logistic and tent maps, $\chi(z)$ is [42]

$$
\begin{aligned}
\text { logistic, } \alpha=4: & \chi(z)=\arcsin ^{2}(\sqrt{z}), \\
\text { tent, } \beta=2: & \chi(z)=\arcsin ^{2}\left[\sin \left(\frac{\pi z}{2}\right)\right],
\end{aligned}
$$

with the resulting expressions for the Hamiltonian deformations:

$$
\begin{aligned}
& f_{\alpha=4}(z)=\sqrt{\frac{1}{z}-1} \arcsin (\sqrt{z}), \\
& f_{\beta=2}(z)=\frac{1}{\pi z} \arcsin \left[\sin \left(\frac{\pi z}{2}\right)\right] \frac{\sqrt{\cos ^{2}\left(\frac{\pi z}{2}\right)}}{\cos \left(\frac{\pi z}{2}\right)} .
\end{aligned}
$$

For both maps, the corresponding eigenvalue is $\lambda=4$.

For the logistic map, it turns out to be possible to obtain an explicit form of the Hamiltonian deformation also for $\alpha=2$,

$$
f(z)=\left(1-\frac{1}{2 z}\right) \log (1-2 z)
$$

\footnotetext{
${ }^{2}$ Equation (10) allows us to continue the tent map to the complex plane: $f(z)=\arcsin (z)$ is expressed through complex logarithms.
} 

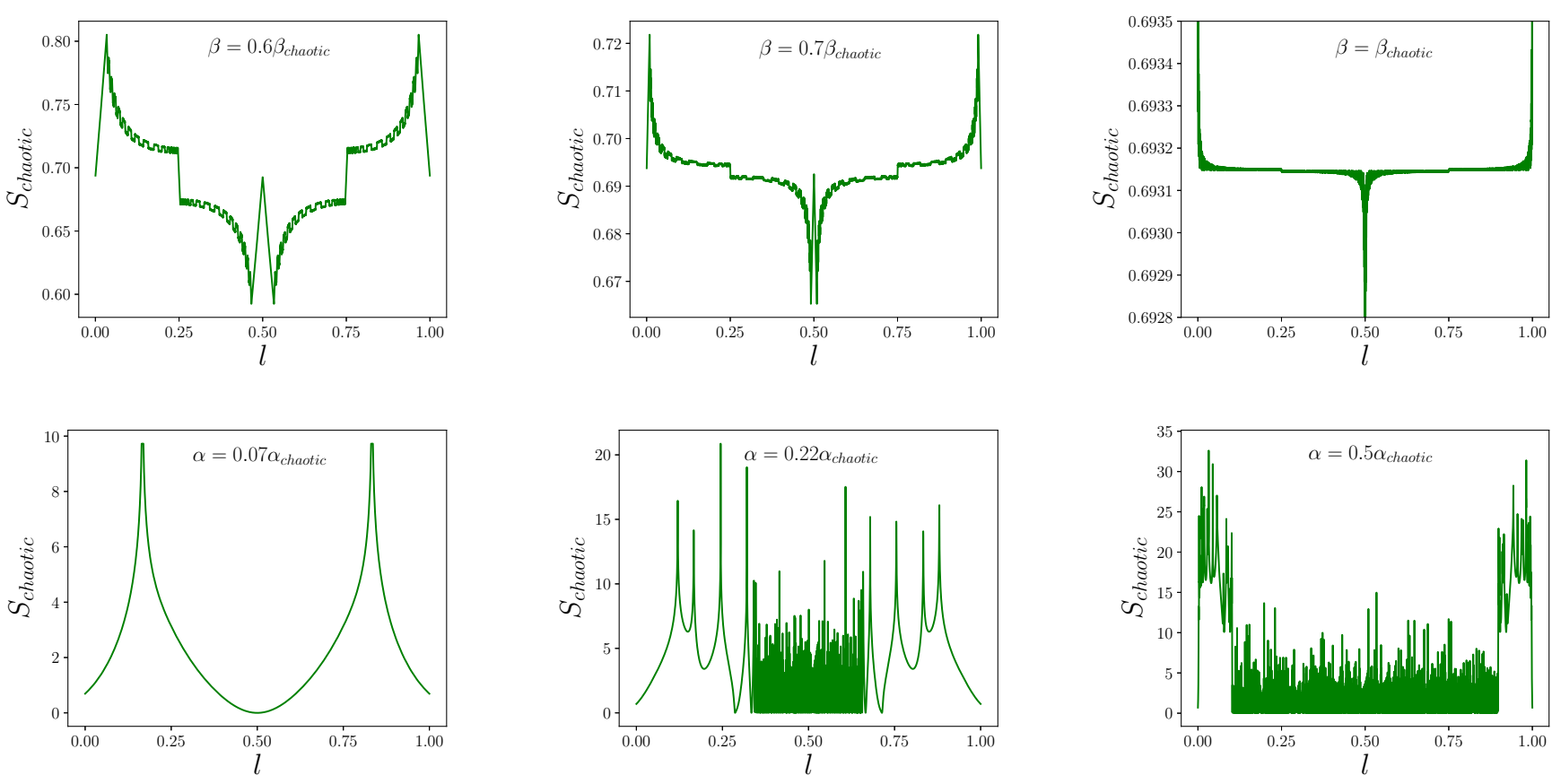

FIG. 1. Irregular part of the entanglement entropy $S_{\text {chaotic }}$ as a function of bipartition coordinate. Note that for the purpose of a nicer representation we define $\Delta S=-\left(S_{\text {reg }}+S_{\text {chaotic }}\right)$, so that in fact the contributions to the entanglement entropy are negative. Top row: After $n=18$ Floquet steps of the tent map. Bottom row: After $n=10$ steps of the logistic map.

as well as an approximate expression at small values of $\alpha$,

$$
f(z)=\frac{(1-z)[1-\alpha z(1-z)]}{(1-2 z)[1-2 \alpha z(1-z)]}
$$

In the Supplemental Material [39], we provide an idea of the derivation and plot profiles of the deformations in the real space domain in the original coordinates, $x=\operatorname{Re}(w)$. One can see that despite the complicated analytic form of expressions (13), the profiles have a rather simple shape that gives hope that this (or a similar) setting allows for experimental implementation.

For the tent map, to reconstruct deformations of the Hamiltonian, one can utilize the expressions corresponding to the logistic map and employ the Milnor-Thurston kneading theory that connects logistic and tent maps by a semiconjugate relation [43] (we outline the basic elements of this construction in the Supplemental Material [39]). Fortunately, within the approach we have taken here, it is possible to proceed without having explicit expressions for $f(z)$.

As we show in the Supplemental Material [39], CFTs with deformations of this type are non-Hermitian. Theories of this kind have attracted interest because of their relevance in the context of nonunitary dynamics of open quantum systems [44], and the entanglement entropy in such models has been studied [45,46]. Experimentally, periodic Floquet driving that induces non-Hermitian evolution can be implemented by switching on and off the coupling between the quantum system and an external bath $[47,48]$.

To derive the real-time dynamics of the CFT, it is natural to get back to the original $w$ coordinates on the strip. The theory is defined on the spatial interval $x \in(0,1)$, so we divide it into two subregions $(0, \ell)$ and $(\ell, 1)$, and compute the evolution of the von Neumann entanglement entropy of this bipartition, which can be expressed as the limit

$$
S(\ell, t)=\lim _{\nu \rightarrow 1} S^{\nu}(\ell, t), \quad S^{\nu}(\ell, t)=\frac{1}{1-v} \log \operatorname{tr}\left[\rho^{\nu}(\ell, t)\right],
$$

where $S^{\nu}(\ell, t)$ is the Renyi entropy. The $\operatorname{trace} \operatorname{tr}\left[\rho^{\nu}(\ell, t)\right]$ can be computed as a one-point correlation function of the primary twist operator $\mathcal{T}_{v}$ of conformal dimension $h$ [49-51]:

$$
\operatorname{tr}\left[\rho^{v}(\ell, t)\right]=\left\langle\psi(t)\left|\mathcal{T}_{v}(\ell)\right| \psi(t)\right\rangle, \quad h=\frac{c}{24}\left(v-\frac{1}{v}\right) .
$$

Here, $|\psi(t)\rangle$ is the state of the system after $n$ cycles of driving $(t=n \tau)$. The explicit expression for this correlation function is [51]

$$
\begin{aligned}
\left\langle\psi(t)\left|\mathcal{T}_{v}(\ell)\right| \psi(t)\right\rangle= & A_{\nu}\left(\frac{\partial z_{n}}{\partial w}\right)^{h}\left(\frac{\partial \bar{z}_{n}}{\partial \bar{w}}\right)^{h}\left(\frac{1}{4 \sqrt{z_{n} \bar{z}_{n}}}\right)^{h} \\
& \times\left(\frac{2 i \epsilon}{\sqrt{z_{n}}-\sqrt{\bar{z}_{n}}}\right)^{2 h}
\end{aligned}
$$

where $\varepsilon$ is a UV cutoff, and $A_{v}$ is a constant that drops out of the subsequent calculations. After taking the $v \rightarrow 1$ limit and subtracting the ground state entanglement and the constant terms, we obtain

$$
\Delta S(\ell, t)=-\frac{c}{12} \log \left(-\frac{\partial_{w} z_{n} \partial_{\bar{w}} \bar{z}_{n}}{\sqrt{z_{n} \bar{z}_{n}}\left(\sqrt{z_{n}}-\sqrt{\bar{z}_{n}}\right)^{2}}\right) .
$$

To extract the fractal contribution to the entropy scaling, it is convenient to use "radial" parametrization $z_{n}=R_{n} \exp \left(i \phi_{n}\right)$ 

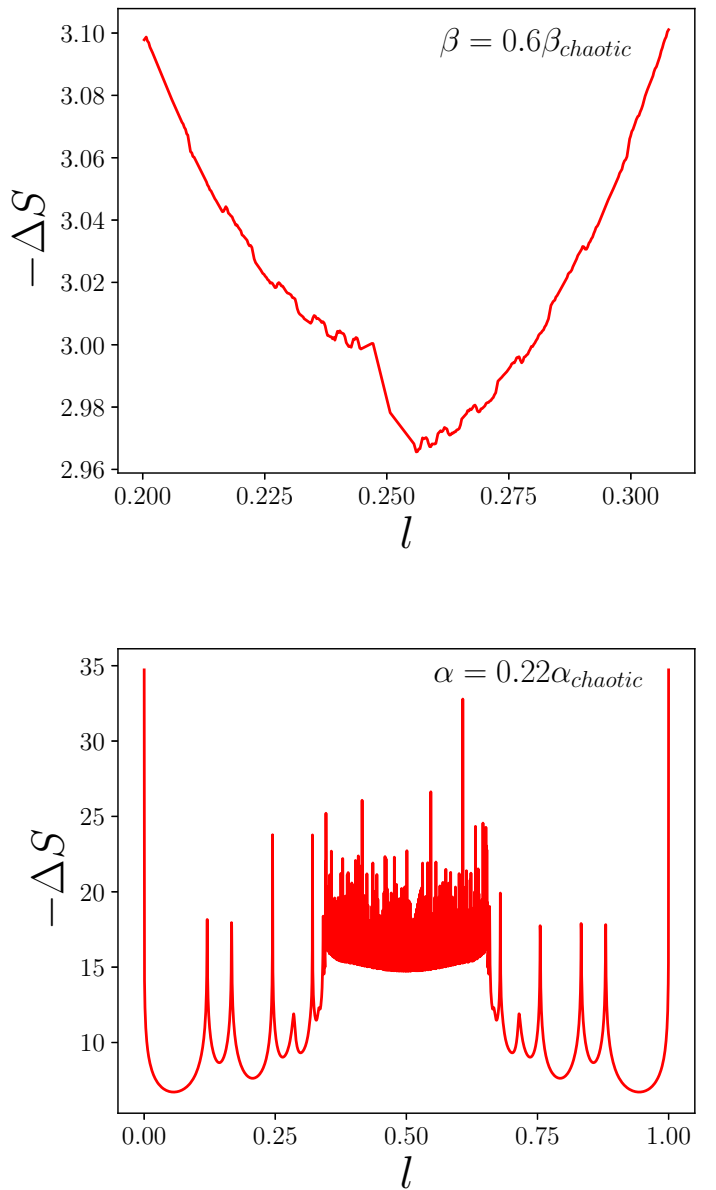

FIG. 2. Full correction to the entanglement entropy $-\Delta S=$ $S_{\text {reg }}+S_{\text {chaotic }}$ (taken with an overall negative sign) as a function of the bipartition coordinate for the tent (top, after $n=18$ driving steps) and the logistic maps (bottom, after $n=10$ driving steps).

and $\bar{z}_{n}=R_{n} \exp \left(-i \phi_{n}\right)$ [9]. In these variables, we get

$$
\begin{aligned}
\Delta S & =-\left(S_{\text {reg }}+S_{\text {chaotic }}\right), \quad S_{\text {reg }}=\log \frac{\partial_{w} z_{n} \partial_{\bar{w}} \bar{z}_{n}}{4 R_{n}^{2}}, \\
S_{\text {chaotic }} & =\log \csc ^{2}\left(\frac{\phi_{n}}{2}\right),
\end{aligned}
$$

where we imposed $c=12$ for the sake of nice normalization. The names "regular" and "chaotic" come from the fact that for both studied types of Floquet driving, $R_{n}$ and the determinant $\partial_{w} z_{n} \partial_{\bar{w}} \bar{z}_{n}$ are regular objects, while $\csc ^{2}\left(\phi_{n} / 2\right)$ that depends on phase $\phi_{n}$ exhibits erratic fractal/chaotic behavior.

For tent map driving, the fractal pattern in $S(\ell, t)$ is explicit, but its contribution to the overall entropy is rather small, especially upon approaching the chaotic regime (top panels of Figs. 1 and 2). Away from $\beta_{\text {chaotic, it has a }}$ shape of a fractal with a rather low Hausdorff dimension. For the logistic map driving, the irregular contribution acquires a much higher amplitude and becomes comparable with the regular part, which allows one to speak about the

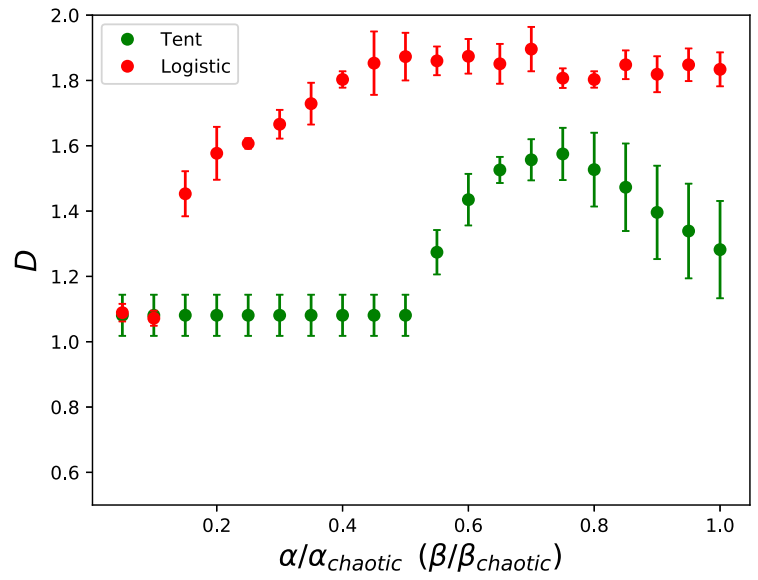

FIG. 3. Dependence of the Hausdorff dimension of $S_{\text {chaotic }}$ on the value of the driving parameter $\alpha / \alpha_{\text {chaotic }}\left(\beta / \beta_{\text {chaotic }}\right)$ for the tent and logistic drivings. For every value of $\alpha(\beta)$, the dimension and the error bars are the correspondingly mean and standard deviations of the so-called local fractal dimensions computed for different windows of spatial scales ranging from $\Delta l \in(0.0004,0.002)$ to $\Delta l \in$ $(0.038,0.04)[39,53]$.

fractal scaling of the entanglement entropy ${ }^{3}$ (bottom panels of Figs. 1 and 2). .

While the self-similarity of the entropy curves is visually recognizable, to really prove that $S_{\text {chaotic }}$ has a fractal shape, it is instructive to compute its Hausdorff dimension. Since here we are dealing with one-dimensional curves which cannot be viewed as sets embedded in a two-dimensional space with both coordinates having the meaning of length, naively applying a box-counting algorithm would lead to meaningless results. Instead, we use the method suggested in Ref. [53] (see also Ref. [54]) designed specifically for computing fractal dimensions of one-dimensional profiles. We describe it in detail in the Supplemental Material [39], and here provide the results. In Fig. 3, we plot the dependence of the fractal dimension of $S_{\text {chaotic }}$. One can see that, in the logistic driving case, significant fractality develops already for small values of $\alpha$, while for the tent map it is not until $\beta=0.5 \beta_{\text {chaotic }}=1$ when the entropy profile acquires fractal features. Error bars reflect how well the estimated value persists across a range of spatial scales. In other words, small error bars mean that the corresponding profile is truly self-similar, while large error bars, such as for $\beta / \beta_{\text {chaotic }}>0.75$, signal that the fractal dimension in fact flows. In both cases, upon $\alpha \rightarrow \alpha_{\text {chaotic }}$ and $\beta \rightarrow \beta_{\text {chaotic }}$, the irregular part of entanglement entropy gradually approaches a fully chaotic regime passing through fractal configurations at smaller values of the parameters. It must be stressed that, for a given choice of $\alpha$ or $\beta$, the entropy profile tends to stabilize as $n \rightarrow \infty$ since the corresponding iterative conformal map (7) stabilizes in this limit.

\footnotetext{
${ }^{3}$ States of a closed CFT obey a strong subadditivity condition, which manifests itself on the level of entanglement entropy scaling as a concavity of $S(l): S^{\prime \prime}(l) \leqslant 0 \forall l$ [52]. Given that our setting is non-Hermitian and can possibly be interpreted as an open quantum system, this constraint can be naturally violated.
} 
This observation is important in two regards. First, for large enough times one can view the evolving stroboscopic state as effectively stationary, making this kind of Floquet driving a candidate mechanism for creating stable in time fractal phases of matter that are not achievable in static systems. Second, the sharp and distinguishable fractal structures at $\alpha \ll \alpha_{\text {chaotic }}$ and $\beta \ll \beta_{\text {chaotic }}$, that could be of higher potential interest than the pseudorandom profiles emerging in the chaotic phase, do not gain "featureless" corrections over the course of the repeated driving.

In this Letter, we have shown that deterministic chaos that emerges in dynamical systems as a result of iterative applications of holomorphic maps can be implemented in the context of Floquet CFT driving. Making use of the fact that the Floquet evolution of a 2D CFT can be expressed as an iterated composition of conformal maps followed by an analytical continuation to real time, we have studied the consequences of choosing the maps that generate dynamical systems exhibiting the order-chaos transition. In the language of Hamiltonian dynamics, it means that the system Hamiltonian is swung between the deformed and undeformed versions at equal periods of time, so that the CFT is perturbed in a stroboscopic manner. When the corresponding dynamical system is tuned towards the transition point, self-similar corrections to the entanglement entropy arise on top of the main smooth component, and the entropy as a function of the bipartition coordinate acquires a fractal shape. Usually, the entropy scales monotonously with the size of the smaller subsystem, and the scaling law serves as a signature of the class to which the quantum state belongs. In this regard, the situation when entanglement entropy strongly oscillates as a function of the number of degrees of freedom included in the subsystem is highly anomalous. It is also interesting to note that, in our setting, the fractal structures emerge as a result of periodic in time regular driving. ${ }^{4}$

\footnotetext{
${ }^{4}$ Recent studies discussed similar phenomena in systems driven randomly or quasiperiodically $[55,56]$.
}

Here, we analyzed the entropy scaling (see the Supplemental Material [39] for the analysis of one-point correlation functions), and such an unusual structure of quantum correlations would definitely affect the transport and spectral properties of the system. Thus it could be important to study states of this kind in more detail because the possibility to create them by means of stroboscopic driving might have unexpected implications for many-body quantum physics.

Since chaotic dynamical systems are ubiquitous, we can expect that quantum states with a nonregular behavior of observables and quantum correlation measures can in fact be engineered in a broad class of Floquet systems. For example, in certain cases $(1+1) \mathrm{D}$ conformal field theories can be regarded as continuous limits of spin chains, which, in turn, can be implemented experimentally using cold atoms in optical lattices [57] or by scanning tunneling microscopy (STM) manipulation [58]. Another potentially promising research avenue is to understand whether fractal-generating driving can be realized as some black-hole perturbations in the holographic context (see, for example, Ref. [59]), and study its meaning in classical and quantum gravity.

We would like to thank P. Caputa for valuable comments on the first version of the manuscript and M. I. Katsnelson, B. Lapierre, and X. Wen for useful discussions. The work of D.S.A., which consisted in designing the project and computing the energy and entanglement evolution for the tent map, was supported by the Russian Science Foundation through Grant No. 17-71-20154 and performed at the Steklov Mathematical Institute of Russian Academy of Sciences. The work of A.A.B., who computed the Hausdorff dimensions of the entropy curves and wrote the manuscript, was supported by the Russian Science Foundation through Grant No. 18-1200185. A.A.I. acknowledges financial support from Dutch Science Foundation NWO/FOM under Grant No. 16PR1024. The work was partially supported by the Knut and Alice Wallenberg Foundation through Grant No. 2018.0060.
[1] L. Jiang, T. Kitagawa, J. Alicea, A. R. Akhmerov, D. Pekker, G. Refael, J. I. Cirac, E. Demler, M. D. Lukin, and P. Zoller, Majorana Fermions in Equilibrium and Driven Cold Atom Quantum Wires, Phys. Rev. Lett. 106, 220402 (2011).

[2] B. J. Wieder, Z. Wang, J. Cano, X. Dai, L. M. Schoop, B. Bradlyn, and B. A. Bernevig, Strong and fragile topological Dirac semimetals with higher-order Fermi arcs, Nat. Commun. 11, 627 (2020).

[3] P. Ponte, Z. Papić, F. Huveneers, and D. A. Abanin, Many-Body Localization in Periodically Driven Systems, Phys. Rev. Lett. 114, 140401 (2015).

[4] D. A. Abanin, W. De Roeck, and F. Huveneers, Theory of many-body localization in periodically driven systems, Ann. Phys. 372, 1 (2016).

[5] M. Maffei, A. Dauphin, F. Cardano, M. Lewenstein, and P. Massignan, Topological characterization of chiral models through their long time dynamics, New J. Phys. 20, 013023 (2018).

[6] L. D'Alessio and M. Rigol, Long-Time Behavior of Isolated Periodically Driven Interacting Lattice Systems, Phys. Rev. X 4, 041048 (2014).

[7] E. A. Stepanov, C. Dutreix, and M. I. Katsnelson, Dynamical and Reversible Control of Topological Spin Textures, Phys. Rev. Lett. 118, 157201 (2017).

[8] H. C. Po, L. Fidkowski, T. Morimoto, A. C. Potter, and A. Vishwanath, Chiral Floquet Phases of Many-Body Localized Bosons, Phys. Rev. X 6, 041070 (2016).

[9] X. Wen and J.-Q. Wu, Floquet conformal field theory, arXiv:1805.00031.

[10] R. Fan, Y. Gu, A. Vishwanath, and X. Wen, Emergent Spatial Structure and Entanglement Localization in Floquet Conformal Field Theory, Phys. Rev. X 10, 031036 (2020). 
[11] B. Lapierre, K. Choo, C. Tauber, A. Tiwari, T. Neupert, and R. Chitra, Emergent black hole dynamics in critical floquet systems, Phys. Rev. Research 2, 023085 (2020).

[12] T. Hikihara and T. Nishino, Connecting distant ends of onedimensional critical systems by a sine-square deformation, Phys. Rev. B 83, 060414(R) (2011).

[13] I. MacCormack, A. Liu, M. Nozaki, and S. Ryu, Holographic duals of inhomogeneous systems: The rainbow chain and the sine-square deformation model, J. Phys. A: Math. Theor. 52, 505401 (2019).

[14] X. Wen, S. Ryu, and A. W. W. Ludwig, Evolution operators in conformal field theories and conformal mappings: Entanglement Hamiltonian, the sine-square deformation, and others, Phys. Rev. B 93, 235119 (2016).

[15] E. Langmann and P. Moosavi, Diffusive Heat Waves in Random Conformal Field Theory, Phys. Rev. Lett. 122, 020201 (2019).

[16] P. Caputa and I. MacCormack, Geometry and complexity of path integrals in inhomogeneous CFTs, J. High Energy Phys. 01 (2021) 027.

[17] P. Moosavi, Inhomogeneous conformal field theory out of equilibrium, arXiv:1912.04821.

[18] K. Gawedzki, E. Langmann, and P. Moosavi, Finite-time universality in nonequilibrium CFT, J. Stat. Phys. 172, 353 (2018).

[19] A. Lazarides, A. Das, and R. Moessner, Periodic Thermodynamics of Isolated Quantum Systems, Phys. Rev. Lett. 112, 150401 (2014)

[20] R. J. Brown, A Modern Introduction to Dynamical Systems (Oxford University Press, Oxford, UK, 2018).

[21] I. Cornfeld, S. Fomin, and Y. Sinai, Ergodic Theory, Vol. 245 (Springer, Berlin, 2012).

[22] A. Katok and B. Hasselblatt, Introduction to the Modern Theory of Dynamical Systems, Vol. 54 (Cambridge University Press, Cambridge, UK, 1997).

[23] D. R. Hofstadter, Energy levels and wave functions of Bloch electrons in rational and irrational magnetic fields, Phys. Rev. B 14, 2239 (1976).

[24] M. Kohmoto, L. P. Kadanoff, and C. Tang, Localization Problem in One Dimension: Mapping and Escape, Phys. Rev. Lett. 50, 1870 (1983).

[25] E. Domany, S. Alexander, D. Bensimon, and L. P. Kadanoff, Solutions to the Schrödinger equation on some fractal lattices, Phys. Rev. B 28, 3110 (1983).

[26] A. F. Beardon, Iteration of Rational Functions (Springer, Berlin, 1991).

[27] J. Shang, Y. Wang, M. Chen, J. Dai, X. Zhou, J. Kuttner, G. Hilt, X. Shao, M. Gottfried, and K. Wu, Assembling molecular Sierpinski triangle fractals, Nat. Chem. 7, 389 (2015).

[28] S. N. Kempkes, M. R. Slot, S. E. Freeney, S. J. M. Zevenhuizen, D. Vanmaekelbergh, I. Swart, and C. Morais Smith, Design and characterization of electrons in a fractal geometry, Nat. Phys. 15, 127 (2018).

[29] S. S. Gubser, C. Jepsen, Z. Ji, and B. Trundy, Continuum limits of sparse coupling patterns, Phys. Rev. D 98, 045009 (2018).

[30] T. Westerhout, E. van Veen, M. I. Katsnelson, and S. Yuan, Plasmon confinement in fractal quantum systems, Phys. Rev. B 97, 205434 (2018).

[31] S. Manna, B. Pal, W. Wang, and A. E. B. Nielsen, Anyons and fractional quantum hall effect in fractal dimensions, Phys. Rev. Research 2, 023401 (2020).
[32] A. A. Iliasov, M. I. Katsnelson, and S. Yuan, Power-law energy level spacing distributions in fractals, Phys. Rev. B 99, 075402 (2019).

[33] A. A. Iliasov, M. I. Katsnelson, and S. Yuan, Hall conductivity of a Sierpinski carpet, Phys. Rev. B 101, 045413 (2020).

[34] A. A. Iliasov, M. I. Katsnelson, and S. Yuan, Linearized spectral decimation in fractals, Phys. Rev. B 102, 075440 (2020).

[35] T. H. Seligman, J. J. M. Verbaarschot, and M. R. Zirnbauer, Quantum Spectra and Transition from Regular to Chaotic Classical Motion, Phys. Rev. Lett. 53, 215 (1984).

[36] T. H. Seligman, J. J. M. Verbaarschot, and M. R. Zirnbauer, Spectral fluctuation properties of Hamiltonian systems: The transition region between order and chaos, J. Phys. A: Math. Gen. 18, 2751 (1985).

[37] S. H. Strogatz, Nonlinear Dynamics and Chaos (AddisonWesley, Boston, 1994).

[38] X. Wen and J. Q. Wu, Quantum dynamics in sine-square deformed conformal field theory: Quench from uniform to nonuniform conformal field theory, Phys. Rev. B 97, 184309 (2018)

[39] See Supplemental Material at http://link.aps.org/supplemental/ 10.1103/PhysRevB.103.L100302 for additional details.

[40] T. Curtright and C. Zachos, Evolution profiles and functional equations, J. Phys. A: Math. Theor. 42, 485208 (2009).

[41] J. W. Milnor, On Lattés maps, arXiv:math/0402147.

[42] P. Collet and J.-P. Eckmann, Iterated Maps on the Interval as Dynamical Systems (Birkhäuser, Boston, 1980).

[43] J. Milnor and W. Thurston, On iterated maps of the interval, in Dynamical Systems (College Park, MD, 1986-87), Lecture Notes in Mathematics Vol. 1342 (Springer, Berlin, 1988), pp. 465-563.

[44] R. Couvreur, J. L. Jacobsen, and H. Saleur, Entanglement in Nonunitary Quantum Critical Spin Chains, Phys. Rev. Lett. 119, 040601 (2017).

[45] D. Bianchini, O. A. Castro-Alvaredo, B. Doyon, E. Levi, and F. Ravanini, Entanglement entropy of non-unitary conformal field theory, J. Phys. A: Math. Theor. 48, 04FT01 (2015).

[46] D. Bianchini and F. Ravanini, Entanglement entropy from corner transfer matrix in Forrester-Baxter non-unitary RSOS models, J. Phys. A: Math. Theor. 49, 154005 (2016).

[47] H. Wu and J. H. An, Floquet topological phases of non-Hermitian systems, Phys. Rev. B 102, 041119(R) (2020).

[48] J. Gong and Q. H. Wang, Stabilizing non-Hermitian systems by periodic driving, Phys. Rev. A 91, 042135 (2015).

[49] P. Calabrese and J. L. Cardy, Entanglement entropy and quantum field theory, J. Stat. Mech. (2004) P06002.

[50] J. L. Cardy, O. A. Castro-Alvaredo, and B. Doyon, Form factors of branch-point twist fields in quantum integrable models and entanglement entropy, J. Stat. Phys. 130, 129 (2008).

[51] P. Calabrese and J. Cardy, Entanglement entropy and conformal field theory, J. Phys. A: Math. Theor. 42, 504005 (2009).

[52] M. Headrick and T. Takayanagi, Holographic proof of the strong subadditivity of entanglement entropy, Phys. Rev. D 76, 106013 (2007). 
[53] B. Dubuc, J. F. Quiniou, C. Roques-Carmes, C. Tricot, and S. W. Zucker, Evaluating the fractal dimension of profiles, Phys. Rev. A 39, 1500 (1989).

[54] I. Pilgrim and R. P. Taylor, Fractal analysis of time-series data sets: Methods and challenges, in Fractal Analysis (IntechOpen, London, 2018).

[55] X. Wen, R. Fan, A. Vishwanath, and Y. Gu, Periodically, Quasiperiodically, and randomly driven conformal field theories: Part I, arXiv:2006.10072.

[56] B. Lapierre, K. Choo, A. Tiwari, C. Tauber, T. Neupert, and R. Chitra, Fine structure of heating in a quasiperiodically driven critical quantum system, Phys. Rev. Research 2, 033461 (2020).

[57] J. J. Garcia-Ripoll, M. A. Martin-Delgado, and J. I. Cirac, Implementation of Spin Hamiltonians in Optical Lattices, Phys. Rev. Lett. 93, 250405 (2004).

[58] C. F. Hirjibehedin, C. P. Lutz, and A. J. Heinrich, Spin coupling in engineered atomic structures, Science 312, 1021 (2006).

[59] T. Andrade, C. Pantelidou, J. Sonner, and B. Withers, Driven black holes: From Kolmogorov scaling to turbulent wakes, arXiv:1912.00032. 